Check for updates

Cite this: RSC Adv., 2019, 9, 22713

\title{
Transforming lignin into porous graphene via direct laser writing for solid-state supercapacitors $\uparrow$
}

\author{
Faisal Mahmood, (DD ad Chi Zhang, ${ }^{\mathrm{b}}$ Yunchao Xie, ${ }^{\mathrm{b}}$ David Stalla, ${ }^{\mathrm{c}}$ Jian Lin (D) ${ }^{\mathrm{b}}$ \\ and Caixia Wan (DD*a
}

Cost-effective valorization of lignin into carbon-based electrode materials remains a challenge. Here we reported a facile and ultrafast laser writing technique to convert lignin into porous graphene as active electrode material for solid-state supercapacitors (SCS). During laser writing, alkaline lignin experienced graphitization. By controlling laser parameters such as power the porous structure and graphitization degree can be well modulated. Graphene obtained at $80 \%$ of laser power setting (LIG-80) had higher graphene quality and more porous structure than that obtained at the lower power levels (i.e., 50\%, 70\%). TEM images revealed that LIG-80 had few-layer graphene structure with fringe-like patterns. LIG-80 proved to be an active electrode material for SCs with a specific capacitance as high as $25.44 \mathrm{mF} \mathrm{cm}^{-2}$ in a $\mathrm{H}_{2} \mathrm{SO}_{4}$ /PVA gel electrolyte, which is comparable or even superior to SCs based on pristine LIG obtained from other carbon precursors. Taken together, our proposed technical route for lignin-based LIG and subsequent application in SCs would not only open a new avenue to lignin valorization, but also produce porous graphene from a renewable carbon precursor for energy storage applications.

Received 29th May 2019

Accepted 7th July 2019

DOI: 10.1039/c9ra04073k

rsc.li/rsc-advances
Given its high carbon content and abundant aromatic subunits, lignin has been explored as a preferred precursor for carbonbased electrode materials. ${ }^{5}$ Lignin-derived electrodes have been prepared mainly via electrospinning and templating methods followed by pyrolysis. ${ }^{6-9}$ The obtained porous carbon may stand out in terms of porosity distribution and energy storage capacities. ${ }^{\mathbf{1 0 , 1 1}}$ However, these methods suffer from complicated fabrication procedures and high energy inputs. Template-free and solvent-free methods can simplify fabrication, but still require prolonged high-temperature treatment, like pyrolysis. ${ }^{12,13}$ The drawbacks with current methods would hinder low cost, mass production of lignin-derived electrode materials. Therefore, a simple, green, and low-cost route for lignin upgrading into carbon-based electrode materials is highly desired.

Among all carbon materials, porous graphitic carbon, such as $3 \mathrm{D}$ porous graphene network, is receiving increasing interests, due to its high electrical conductivity, good thermal stability, and high specific surface area. ${ }^{\mathbf{1 4 , 1 5}}$ The general strategies are graphene oxide (GO) reduction and chemical vapor deposition (CVD), but these techniques rely on either corrosive multistep reactions or energy-intensive thermal treatment, which limits their large-scale application. Recently direct laser writing (DLW) technique is emerging as a facile and scalable process for porous graphene production. ${ }^{\mathbf{1 6 - 1 8}}$ It uses a commercial infrared $\mathrm{CO}_{2}$ laser as an energy source to convert carbon precursors to porous graphene. Laser-induced graphene (LIG) exhibits hierarchical porous structure with large surface area, excellent conductivity, and high thermal stability. ${ }^{16}$ DLW is a Department of Biomedical, Biological, and Chemical Engineering, University of 5650; Tel: +15738847882

${ }^{b}$ Department of Mechanical and Aerospace Engineering, University of Missouri, Columbia 65211, USA

${ }^{c}$ Electron Microscopy Core, University of Missouri, Columbia 65211, USA

${ }^{d}$ Department of Energy Systems Engineering, University of Agriculture Faisalabad, Faisalabad 38000, Pakistan

$\dagger$ Electronic supplementary information (ESI) available. See DOI: 10.1039/c9ra04073k 
a one-step, energy-saving, chemical-free process conducted under ambient temperature, which overcomes the limitations of the aforementioned GO reduction and CVD methods. Moreover, it allows for roll-to-roll manufacturing, thus enabling large-scale fabrication of graphitic carbon. LIG has been used for various applications, such as supercapacitors, microfluidic devices, catalysts, and pressure sensors. ${ }^{\mathbf{1 4 , 1 9 , 2 0}}$ LIG-based SCs were shown to have high capacitance due to hierarchical porous structures and high conductivity. ${ }^{16,21}$ They offer more energy or power density or both than commercial aluminum electrolytic capacitors, thin film lithium-ion batteries, and activated-carbon supercapacitors. ${ }^{\mathbf{1 6}}$

Laser writing/irradiation induces localized high temperature and pressure, which provides unique reaction conditions for graphene formation. ${ }^{22}$ LIG properties, including porosity, surface chemistry, and composition, can be tuned by regulating laser parameters (e.g., power, scan rate) and precursor composition/ structure. ${ }^{14}$ Among laser parameters, laser power plays a key role in tailoring morphology and structure of LIG. $^{\mathbf{1 6}}$ In general, a threshold power level is required for the induction of graphene formation, and higher power level above the threshold leads to higher graphene quality. However, too strong laser power can damage graphene structure and even ablate carbon precursors. In terms of carbon precursor, DLW can be applied to both synthetic polymers and naturally occurring materials for graphene formation. ${ }^{23}$ Compared to synthetic polymers, biomass is an attractive precursor due to its renewability, abundance, and low cost. Lignin as a major constituent in biomass was shown to make significant contribution to LIG formation, and biomass with higher lignin contents favors graphene formation. ${ }^{23,24} \mathrm{~A}$ recent prior study also showed that LIG can be formed from alkaline lignin, a kind of technical lignin extracted from biomass, and subsequent on-chip interdigitated microsupercapacitors (MSCs) had similar capacitance to those made from polyimide (PI)-based LIG electrode. ${ }^{25}$ Given aromatic structure and abundant availability, technical lignin warrants further exploration for LIG formation toward energy storage application. Such implementation would enable scalable, cost-effective lignin upgrading into high-value graphene-based carbon materials and highperformance energy storage devices.

In this study, we focused on upgrading lignin into LIG and further assembling it into solid-state SCs with high electrochemical performance. Lignin film composed of alkaline lignin and PEO (serving as a binder for film fabrication) was used as precursor for LIG production. Different laser powers were applied to induce graphene from lignin. Obtained LIG was characterized for structural and chemical properties via a series of analytical techniques. Finally, sandwiched SCs were fabricated using lignin-based LIG electrodes and $\mathrm{H}_{2} \mathrm{SO}_{4} / \mathrm{PVA}$ gel electrolyte, and evaluated for their electrochemical performance.

\section{Materials and methods}

\subsection{Lignin film preparation}

Water-soluble softwood alkaline lignin (AL) (TCI America ${ }^{\mathrm{TM}}$, Mfr No. L0082500G) was purchased from Fisher Scientific
(Hampton, NH, USA) and used as received. Below is the procedure for lignin film fabrication. First, $0.75 \mathrm{~g}$ of PEO (Polyox ${ }^{\mathrm{TM}}$ WSR 301, $M_{\mathrm{W}}=4 \times 10^{6}$ ) was mixed with $20 \mathrm{~mL}$ of water, and then continuously stirred at room temperature until a clear, viscous PEO solution was formed. Thereafter, $1.5 \mathrm{~g}$ of AL was added to the as-prepared $20 \mathrm{~mL}$ of PEO solution. After stirring overnight, a viscous, dark brown solution was obtained, which was further cast onto a $9 \mathrm{~cm}$ plastic Petri dish for the film formation. After drying in open air, lignin film comprising $\mathrm{AL}$ and PEO with a weight mixing ratio of $2: 1$ was peeled off from the Petri dish and then cut into desired dimensions. All the films were stored in covered Petri dish in room temperature prior to use. A representative AL/PEO film is shown in Fig. $1 \mathrm{~b}$.

\subsection{Laser writing}

Lignin films were first attached to a stage by a removable double-sided Scotch tape in order to avoid the deformation of films due to drastically increased temperature during laser writing. A $10.6 \mu \mathrm{m} \mathrm{CO}_{2}$ laser (H-Series $20 \times 12$ Desktop laser, Full Spectrum, Les Vegas, NV) with an upper power of $40 \mathrm{~W}$ and a pulse duration of $\sim 14 \mu \mathrm{s}$ was used for scribing lignin film. All the scribing tests were performed under ambient conditions with a beam size of $\sim 100 \mu \mathrm{m}$, a scan rate of $20 \mathrm{~cm} \mathrm{~s}^{-1}$, and an image density of 1000 pulses per inch (PPI). Laser power effects were studied by applying $50-90 \%$ of $40 \mathrm{~W}$ laser power setting. Scribed lignin film was denoted as LIG-X, where $\mathrm{X}$ stands for power percentage of the laser.

\subsection{SCs fabrication}

Lignin-based LIG was used as both electrode and current collector for SCs. The following procedure was followed to assemble LIG electrode. Silver paint (Pellco®, Catalog No. 16034, Ted Pella, Redding, CA) was applied to the edge of LIG (1 $\mathrm{cm}^{2}$ ) embedded on the lignin film for better electrical connection and extended with conductive copper tapes. A Kapton® PI tape was employed on the silver paint and portion of the copper tape to avoid their contact with electrolyte. After assembly, one LIG electrode was coated with $1.0 \mathrm{M}$ PVA/ $\mathrm{H}_{2} \mathrm{SO}_{4}$ gel electrolyte and then stacked with another electrode, forming a sandwiched $\mathrm{SC}$, as depicted in Fig. 1a. $\mathrm{PVA} / \mathrm{H}_{2} \mathrm{SO}_{4}$ gel electrolyte was prepared by mixing $1.0 \mathrm{~g}$ of $\mathrm{H}_{2} \mathrm{SO}_{4}$ and $1.0 \mathrm{~g}$ of PVA $\left(M_{\mathrm{W}}=\right.$ 124 000-186 000) in $10 \mathrm{~g}$ of $\mathrm{H}_{2} \mathrm{O}$ until a clear gel was formed. Fabricated SCs were placed in vacuum desiccator to allow gel electrolyte to become completely dry. After device fabrication, the conductive copper tape was attached to an electrochemical workstation for electrochemical analysis.

\subsection{Characterization}

Morphology of LIG was examined using FEI Quanta 600 FEG environmental scanning electron microscope (ESEM) equipped with a Bruker Quantax 200 Silicon Drift Detector and operated at $15 \mathrm{kV}$ and $100 \mathrm{pA}$. Scribed lignin films were cut into small dimensions and mounted onto an adhesive SEM sample holder for imaging. The thickness of LIG was measured via SEM imaging of cross-section of scribed lignin film. High resolution transmission electron microscopy (HRTEM) was used to exam 


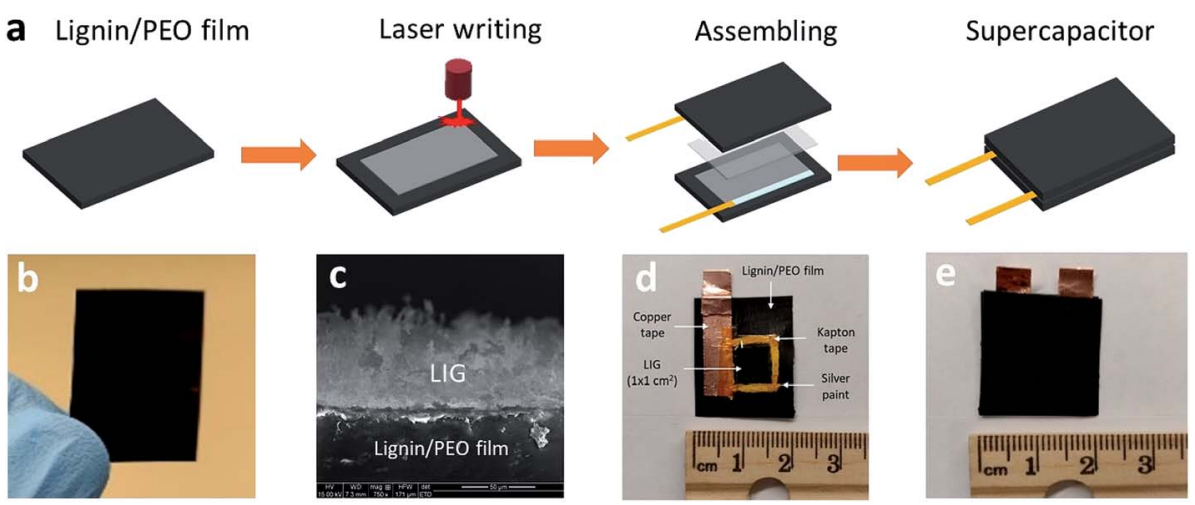

Fig. 1 Process of converting lignin to LIG and subsequent SC fabrication. (a) Overview of the DLW process and device fabrication. (b) An AL/PEO film. (c) Cross-sectional SEM image of LIG-80 (scale bar is $50 \mu \mathrm{m}$, showing LIG thickness of $\sim 53 \mu \mathrm{m}$ ). (d) Assembled single electrode, with $1 \mathrm{M}$ $\mathrm{H}_{2} \mathrm{SO}_{4}$ /PVA gel electrolyte applied. (e) Fully assembled sandwich-like SC using two LIG electrodes derived from lignin.

microstructure and nanostructure of LIG. TEM images were taken on FEI Tecnai F30 Twin $300 \mathrm{kV}$ TEM. LIG powder was scrapped from scribed lignin film and sonicated for $5 \mathrm{~min}$ in ethanol before being transferred onto a C-flat TEM grid for imaging. Raman spectra were acquired on a Renishaw inVia ${ }^{\mathrm{TM}}$ Raman Spectrometer at $633 \mathrm{~nm}$. X-ray diffraction (XRD) patterns were acquired on a Scintag X2 diffractometer equipped with a $\mathrm{Cu} \mathrm{K} \alpha$ radiation (Scintag, Inc., Cupertino, CA). Survey scans were conducted at $0.02^{\circ}$ step size with a scanning rate of 6 $\mathrm{s} / 0.02^{\circ}$, while detailed peak analyses were conducted at the same step size but with a scanning rate of $12 \mathrm{~s} / 0.02^{\circ}$. X-ray photoelectron spectra were acquired on a Kratos Axis 165 Photoelectron spectroscopy system with a vacuum level of $3.0 \times$ $10^{-8}$ Torr. Survey spectra were recorded in a step size of $0.5 \mathrm{eV}$ with pass energy of $160 \mathrm{eV}$, while high resolution elemental spectra were recorded in $0.1 \mathrm{eV}$ with pass energy of $20 \mathrm{eV}$. All the spectra were corrected using the $\mathrm{C}$ 1s peak $(284.4 \mathrm{eV})$ as reference.

Based on Raman spectra data, the crystalline size of graphitic carbon in the $a$ axis $\left(L_{a}\right)$ was calculated from the intensity ratio of $\mathrm{G}$ peak $\left(I_{\mathrm{G}}\right)$ and D peak $\left(I_{\mathrm{D}}\right)$ following eqn $(1):^{26}$

$$
L_{a}=\left(2.4 \times 10^{-10}\right) \times \lambda_{l}{ }^{4} \times\left(\frac{I_{\mathrm{G}}}{I_{\mathrm{D}}}\right)
$$

where $\lambda_{1}$ is the wavelength of the Raman laser $\left(\lambda_{1}=633 \mathrm{~nm}\right)$.

\subsection{Electrochemical analysis}

Cyclic voltammetry (CV) and galvanostatic charge-discharge (CD) measurements were conducted on CHI 660D electrochemical workstation (CHI Instruments, USA). The CV tests were carried out at an operating voltage $(0-1.0 \mathrm{~V})$ with different scan rates ranging from 10 to $500 \mathrm{mV} \mathrm{s}^{-1}$. The CD tests were performed with current densities in a range of $0.1-1.0 \mathrm{~mA} \mathrm{~cm} \mathrm{~cm}^{-2}$. Unlike conventional SCs whose performance was evaluated based on mass of active materials, the proposed SCs, similar to MSCs, have much less mass of active materials and thus their footprint area becomes the key consideration. ${ }^{27}$ Here areal capacitance $\left(C_{\mathrm{A}}\right)$ and volumetric capacitance $\left(C_{\mathrm{V}}\right)$ were calculated, which was further used for the calculation of spatial energy and power densities.

The specific areal capacitance $\left(C_{\mathrm{A}}\right.$, in $\left.\mathrm{mF} \mathrm{cm}^{-2}\right)$ based on $\mathrm{CV}$ curves was calculated following eqn (2) below:

$$
C_{\mathrm{A}}=\frac{\int_{V_{\mathrm{i}}}^{V_{\mathrm{f}}} I \mathrm{~d} V}{2 \times S \times \nu \times\left(V_{\mathrm{f}}-V_{\mathrm{i}}\right)}
$$

where $S$ is the surface area (in $\mathrm{cm}^{2}$ ) of an active LIG electrode, with $1 \mathrm{~cm}^{2}$ for the device configuration in this work; $\nu$ is the voltage scan rate (in $\mathrm{V} \mathrm{s}^{-1}$ ); $V_{\mathrm{f}}$ and $V_{\mathrm{i}}$ are the potential limits used for the $\mathrm{CV}$ analysis (in V); $I$ is the voltammetry current (in A); and $\int_{V_{\mathrm{i}}}^{V_{\mathrm{f}}} I \mathrm{~d} V$ denotes the integrated area of CV curve.

The $C_{\mathrm{A}}$ (in $\mathrm{mF} \mathrm{cm}^{-2}$ ) based on the CD curves was calculated using eqn (3):

$$
C_{\mathrm{A}}=\frac{I}{S \times\left(\frac{\mathrm{d} V}{\mathrm{~d} t}\right)}
$$

where $I$ is the discharge current (in A); $S$ is the surface area of the LIG $\left(\mathrm{cm}^{2}\right)$, with $1 \mathrm{~cm}^{2}$ for the device configuration here; and $\mathrm{d} V /$ $\mathrm{d} t$ is the slope of the galvanostatic discharge curves.

The volumetric capacitance $\left(C_{\mathrm{V}}\right)$ (in $\mathrm{mF} \mathrm{cm}^{-3}$ ) based on either $\mathrm{CV}$ or CD curves was calculated by dividing $C_{\mathrm{A}}$ by the thickness of active material $(d$, in $\mathrm{cm})$ following eqn (4):

$$
C_{\mathrm{V}}=\frac{C_{\mathrm{A}}}{d}
$$

The specific areal energy $\left(E_{\mathrm{A}}\right.$ in $\left.\mathrm{mW} \mathrm{h} \mathrm{cm}{ }^{-2}\right)$ and power $\left(P_{\mathrm{A}}\right.$, in $\mathrm{mW} \mathrm{cm}^{-2}$ ) densities were calculated by eqn (5) and (6), respectively. For the specific volumetric energy $\left(E_{\mathrm{V}}\right.$, in $\mathrm{mW} \mathrm{h}$ $\left.\mathrm{cm}^{-3}\right)$ and power $\left(P_{\mathrm{V}}\right.$, in $\left.\mathrm{mW} \mathrm{cm}^{-3}\right)$ densities, eqn (7) and (8) were used for the calculations, respectively.

$$
\begin{gathered}
E_{\mathrm{A}}=\frac{1}{2} \times C_{\mathrm{A}} \times \frac{(\Delta V)^{2}}{3600} \\
P_{\mathrm{A}}=\frac{E_{\mathrm{A}}}{\Delta t} \times 3600
\end{gathered}
$$




$$
\begin{gathered}
E_{\mathrm{V}}=\frac{1}{2} \times C_{\mathrm{V}} \times \frac{(\Delta V)^{2}}{3600} \\
P_{\mathrm{V}}=\frac{E_{\mathrm{V}}}{\Delta t} \times 3600
\end{gathered}
$$

where $C_{\mathrm{A}}$ and $C_{\mathrm{V}}$ are based on CD curves and calculated by eqn (3) and (4), respectively; $\Delta V=V_{\max }-V_{\text {drop }}$ is equivalent to the discharge potential range $\left(V_{\max }\right.$ is the voltage of $1 \mathrm{~V}$ for $\mathrm{H}_{2} \mathrm{SO}_{4}$ / PVA gel electrolyte); $V_{\text {drop }}$ is the voltage drop indicated from the difference of the first two points in the data obtained from the discharge curves; and $\Delta t$ is the discharge time (in s).

\section{Results and discussion}

\subsection{Characterization of lignin-derived LIG}

Lignin-derived LIG film was characterized for its morphology and structural properties by ESEM and HRTEM. The morphology of the lignin film irradiated with $80 \%$ laser power setting (denoted as LIG-80) was depicted in Fig. 2a-c. It showed a typical porous foam-like structure with numerous macrospores, which is very similar to that of LIG derived from PI (Fig. 2a). ${ }^{16}$ The cross-sectional SEM image of laser-irradiated lignin film showed a porous layer with $\sim 53 \mu \mathrm{m}$ thickness embedded on the film (Fig. 1c). SEM images at high magnification also showed beehive-like nanoporous structure with a mixture of mesopores and macropores in the carbon matrix
(Fig. $2 \mathrm{c}$ and $\mathrm{S} 1 \dagger$ ). The highly porous structure was caused by the rapid liberation of gaseous molecules (e.g., $\left.\mathrm{CO}_{2}, \mathrm{H}_{2} \mathrm{O}\right)$ resulting from the degradation of PEO and partial decomposition of lignin upon laser irradiation. ${ }^{28}$ On the other hand, pure PEO film cannot be induced into graphene upon laser irradiation. Since laser irradiation is a photothermal treatment in principle, laser intensity/power is a key variable for inducing graphene from carbon precursor. ${ }^{16,24}$ By applying a wide range of laser power (50-90\%), we found that morphology and porous structure of LIG evolved with the change in power level (Fig. S2 $\dagger$ ). Laser writing at $50 \%$ laser power or above opened up pores of the film, and a higher power level led to more porous structures. However, when the power level reached $90 \%$, it had deleterious effects, and the cracks on the film were visible due to the severity of photothermal treatment as previously reported. ${ }^{24}$ Taken together, laser irradiation with an optimal power range led to the formation of hierarchical porous carbon from lignin, particularly graphene carbon as identified below. Such hierarchical porous structure is beneficial for electrochemical performance, as it can provide enhanced accessible surface area and facilitate fast diffusion of electrolyte into electrode. ${ }^{29,30}$

More detailed structures of lignin-derived carbon were characterized by TEM (Fig. 2d-f). HRTEM image of LIG-80 displayed graphene fringes with a characteristic of $0.335 \mathrm{~nm} d$ spacing, corresponding to the gap between two adjacent (002) planes in graphitic carbon (Fig. 2f). Graphene was also shown to
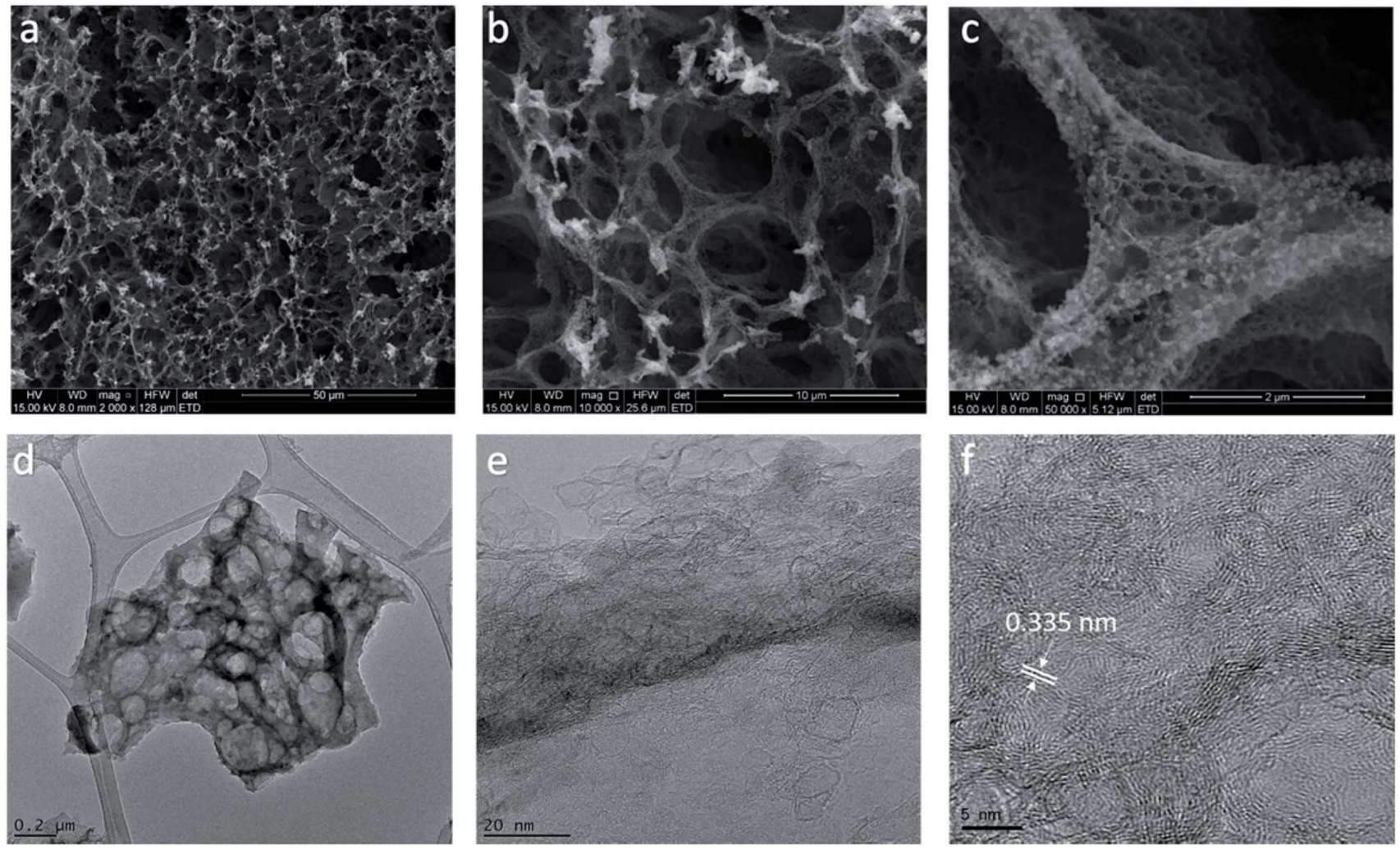

Fig. 2 Morphology and structure of LIG-80. (a) SEM image; scale bar is $50 \mu \mathrm{m}$. (b and c) High resolution SEM images; scale bars are $10 \mu \mathrm{m}$ and 2 $\mu \mathrm{m}$, respectively. (d) TEM image; scale bar is $0.2 \mu \mathrm{m}$. (e and f) HRTEM images; scale bars are $20 \mathrm{~nm}$ and $5 \mathrm{~nm}$, respectively. LIG embedded on the lignin/PEO film was used for SEM imaging, while LIG powder scraped from the lignin/PEO film was used for TEM imaging. 
have thin flakes with few-layer structures (Fig. 2f). Moreover, graphitic carbon formed at a higher laser power level exhibited more ordered structure (Fig. S3† and 2e). Porous graphene network with abundant defect-rich boundaries as mentioned below is suggested to have improved electrochemical capacitance compared to defect-free graphene structure. ${ }^{16}$

The presence of graphene in lignin-based LIG is also evidenced by Raman spectra, which showed three characteristic peaks of graphene, including a D peak at $\approx 1350 \mathrm{~cm}^{-1}$, a G peak at $\approx 1580$, and a $2 \mathrm{D}$ peak at $\approx 2670 \mathrm{~cm}^{-1}$ (Fig. 3 ). The $\mathrm{D}$ peak is suggestive of defects or bent $\mathrm{sp}^{2}$-carbon bonds, whereas $\mathrm{G}$ peak is the first-order Raman band of all $\mathrm{sp}^{2}$ hybridized carbon. ${ }^{31}$ The 2D peak originates from second order zone boundary phonons. ${ }^{31}$ Compared to LIG obtained from the other two power levels (i.e., LIG-50, LIG-70), LIG-80 had the much stronger peak intensities. It also had the highest $I_{\mathrm{G}} / I_{\mathrm{D}}$ ratio, with the average crystalline size calculated to be $41 \mathrm{~nm}$. Moreover, LIG-80 had the highest $I_{2 \mathrm{D}} / I_{\mathrm{G}}$ ratio, indicating the fewest number of graphene layers. ${ }^{24}$ Although the $\mathrm{D}$ peak was not suppressed at a higher power (likely due to the presence of PEO in the lignin film), Raman spectra results suggested that by increasing the laser intensity from $50 \%$ to $80 \%$ power, higher quality graphene with more crystalline structure and less stacked layers can be generated from alkaline lignin.

Graphene formed in LIG-80 was further confirmed by X-ray diffraction (XRD) (Fig. S4†). The peak at $2 \theta \approx 24.9^{\circ}$ was assigned to (002) reflection (inset in Fig. S4†), indicating a $d$ spacing of $0.357 \mathrm{~nm}$. This characteristic peak shifted to one degree lower than that reported for LIG derived from PI. ${ }^{\mathbf{1 6}}$ This suggested larger interlayer spacing in the lignin-based LIG, which could be due to higher oxidation of lignin/PEO film upon laser irradiation. The slight disparity between XRD and HRTEM results also suggested that interlayer spacing can vary slightly from particle to particle (Fig. S4 $\uparrow$ and 2f). Nevertheless, the value of $d$-spacing obtained here was comparable to that

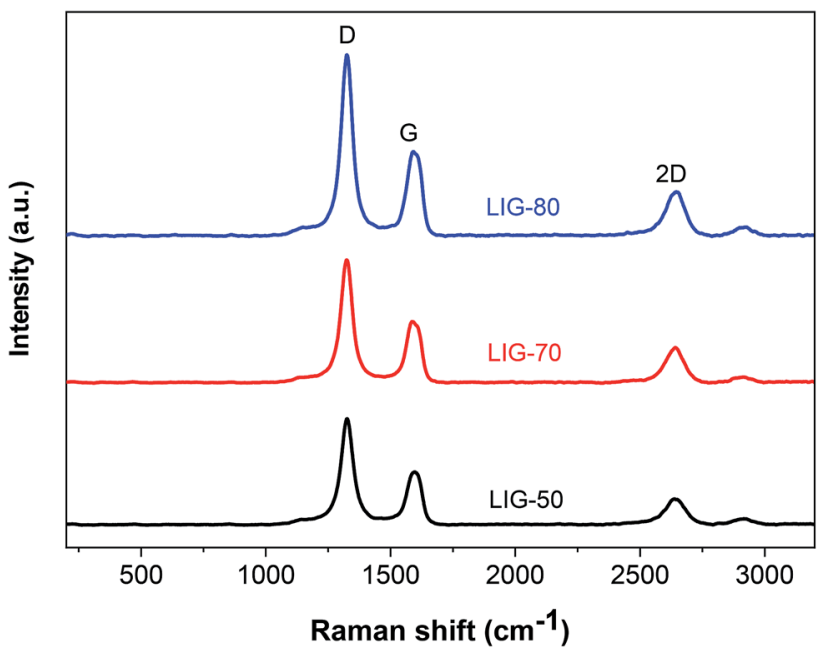

Fig. 3 Raman spectra of LIG showing three prominent peaks (i.e., D, G, and 2D). The pristine lignin/PEO film showed no signals via Raman scan. LIG embedded on the lignin/PEO film was used for Raman spectroscopy. reported for laser writing to generate graphene from graphite oxide. $^{32}$ Overall, the XRD results supported that lignin was successfully transformed to graphene.

XPS spectra show that LIG-80 had significantly increased carbon content compared to the pristine (non-irradiated) lignin/PEO film (Fig. S5 $\dagger$ ). LIG-80 also contained small amounts of impurities (e.g., $\mathrm{Na}, \mathrm{S}$ ) originating from alkaline lignin. The content of Na was significantly reduced by laser irradiation, suggesting that lasing would induce combined photothermal and photochemical reactions. High resolution $\mathrm{C}$ 1s XPS spectra revealed four dominant peaks: $\mathrm{sp}^{2} \mathrm{C}-\mathrm{C}$ bond at $\sim 284.4 \mathrm{eV}, \mathrm{C}-\mathrm{O}$ bond at $\sim 285.7 \mathrm{eV}, \mathrm{C}=\mathrm{O}$ bond at $\sim 288.5 \mathrm{eV}$, and $\mathrm{O}-\mathrm{C}=\mathrm{O}$ bond at $\sim 290.5 \mathrm{eV}$ (Fig. 4a). The $\mathrm{sp}^{2} \mathrm{C}-\mathrm{C}$ peak became dominant in LIG-80, accounting for $73.1 \%$ of $\mathrm{C} 1 \mathrm{~s}$ major functional groups (Fig. 4b). The $\mathrm{O}-\mathrm{C}=\mathrm{O}$ peak disappeared after laser irradiation. The content of $\mathrm{C}-\mathrm{C}$ functionality in LIG-80 is comparable to that in LIG obtained from other biomass-based precursors (e.g., wood, nanocellulose, lignin/ PVA film). ${ }^{23,24,33}$ Compared to PI film, biomass-based precursors have a lower degree of carbon enrichment and oxygen suppression upon laser irradiation, which would be attributed to highly functionalized structure of biomass or lignin. Nevertheless, the significant increase in $\mathrm{C}-\mathrm{C}$ bond and decrease in $\mathrm{C}-\mathrm{O}$ bond confirmed that lignin experienced carbonization and graphitization during laser scribing. As a result, LIG was dominated by $\mathrm{sp}^{2}$ carbon, which agreed well with the Raman and XRD results.

\subsection{Electrochemical performance of lignin-based LIG}

The above results indicated that laser writing facilely transformed lignin into hierarchical porous graphene. All the characteristics suggested that derived LIG has great promise for high-performance active electrodes. Here we assembled sandwiched SCs using lignin-based LIG as both electrode and current collector. The device architecture was depicted in Fig. 1a. The electrochemical performance of the fabricated LIGSCs was evaluated based on their CV and CD measurements. The CV curves of LIG-80 at various scan rates $\left(10-500 \mathrm{mV} \mathrm{s}^{-1}\right)$ showed pseudo-rectangular shape, indicating good double-layer capacitive behaviors (Fig. $5 \mathrm{a}$ and b). The $C_{\mathrm{A}}$ of LIG-80 as a function of scan rate was shown in Fig. $5 \mathrm{c}$. At 10 and $20 \mathrm{mV} \mathrm{s}^{-1}$ scan rates, the SC had 11.64 and $8.84 \mathrm{mF} \mathrm{cm}^{-2} C_{\mathrm{A}}$, respectively. These $C_{\mathrm{A}}$ values were almost double that obtained from SCs based on pristine LIG from PI film and aqueous $1 \mathrm{M} \mathrm{H}_{2} \mathrm{SO}_{4}$ electrolyte (e.g., $\sim 4.3 \mathrm{mF} \mathrm{cm}{ }^{-2}$ at $\left.20 \mathrm{mV} \mathrm{s}^{-1}\right) .{ }^{16}$ When the scan rate was increased to $500 \mathrm{mV} \mathrm{s}^{-1}$, the $C_{\mathrm{A}}$ still remained to be $1.59 \mathrm{mF} \mathrm{cm}^{-2}$. In contrast to LIG-80 electrode, the other two electrodes (i.e., LIG-50, LIG-70) also showed near pseudorectangular shape (Fig. S6a and $\mathrm{b} \dagger$ ), but had much lower capacitance (Fig. S6c $\dagger$ ), which was also evidenced by their CV curves (Fig. S6d and $\mathrm{e}^{\dagger}$ ). Significantly higher electrochemical performance of LIG-80 electrode could be attributed to its abundant wrinkle-/fringe-like graphene structure as revealed by HRTEM images (Fig. 2f).

The charge/discharge (CD) curves of LIG-80 at different current densities $\left(0.1-1.0 \mathrm{~mA} \mathrm{~cm}^{-1}\right)$ are depicted in Fig. $5 \mathrm{~d}$. The 

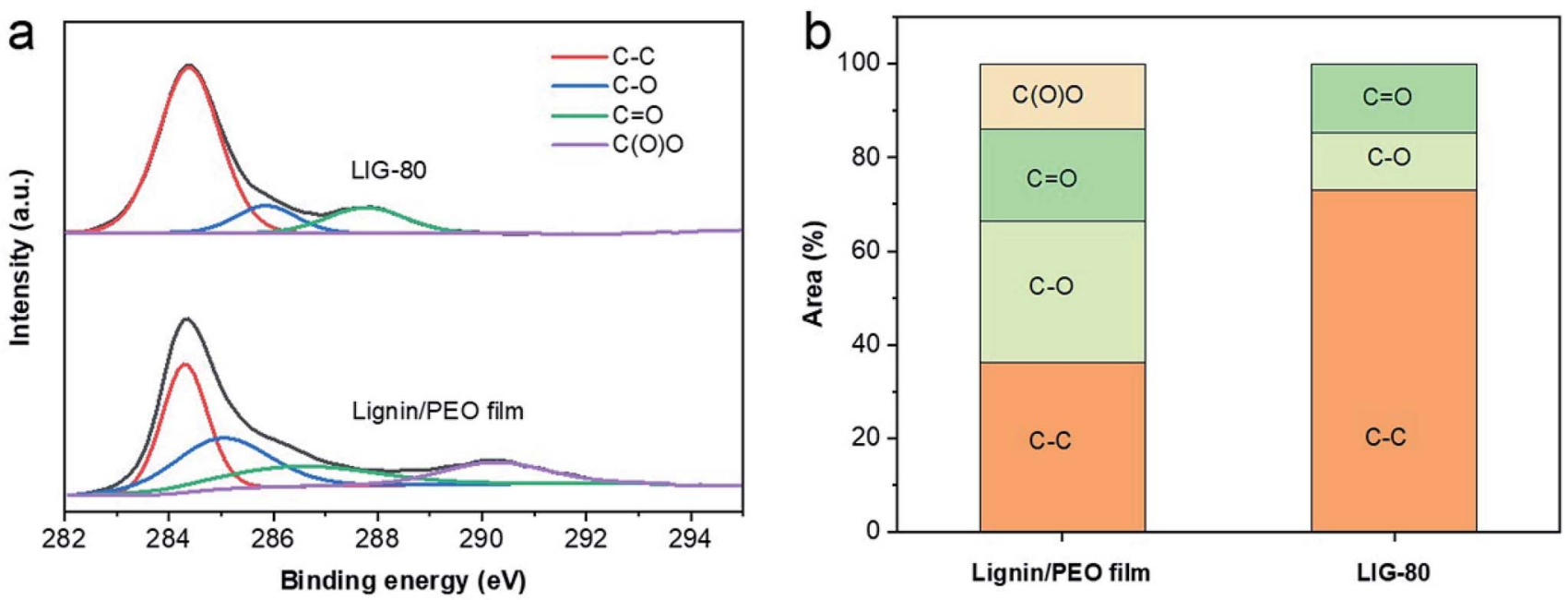

Fig. 4 XPS spectra. (a) High resolution C 1s XPS spectra. (b) Functionality revealed by high resolution C 1s XPS. LIG embedded on the lignin/PEO film was used for XPS spectroscopy, and the pristine lignin/PEO film used as a control.

CD curves were nearly triangle in shape, proving good capacitive behavior. From the initial stage of discharge, the voltage drop indicates slight internal resistance of the SC. The specific capacitance $\left(C_{\mathrm{A}}\right)$ as a function of current density is presented in Fig. 5e. The $C_{\mathrm{A}}$ was $25.44 \mathrm{mF} \mathrm{cm}^{-2}$ at a current density of $0.1 \mathrm{~mA}$ $\mathrm{cm}^{-2}$, with the corresponding specific volumetric capacitance
$\left(C_{\mathrm{V}}\right)$ of $4.80 \mathrm{~F} \mathrm{~cm}^{-3}$ (Fig. S7b $\dagger$ ). It decreased gradually with increasing discharge current densities, but still delivered 9.36 and $4.38 \mathrm{mF} \mathrm{cm}^{-2}$ at the current densities of 0.5 and $1.0 \mathrm{~mA}$ $\mathrm{cm}^{-2}$, respectively. These values were comparable or even higher than those reported for SCs based on pristine LIG and some other carbon at the same current densities. ${ }^{16,25,34-38}$ For
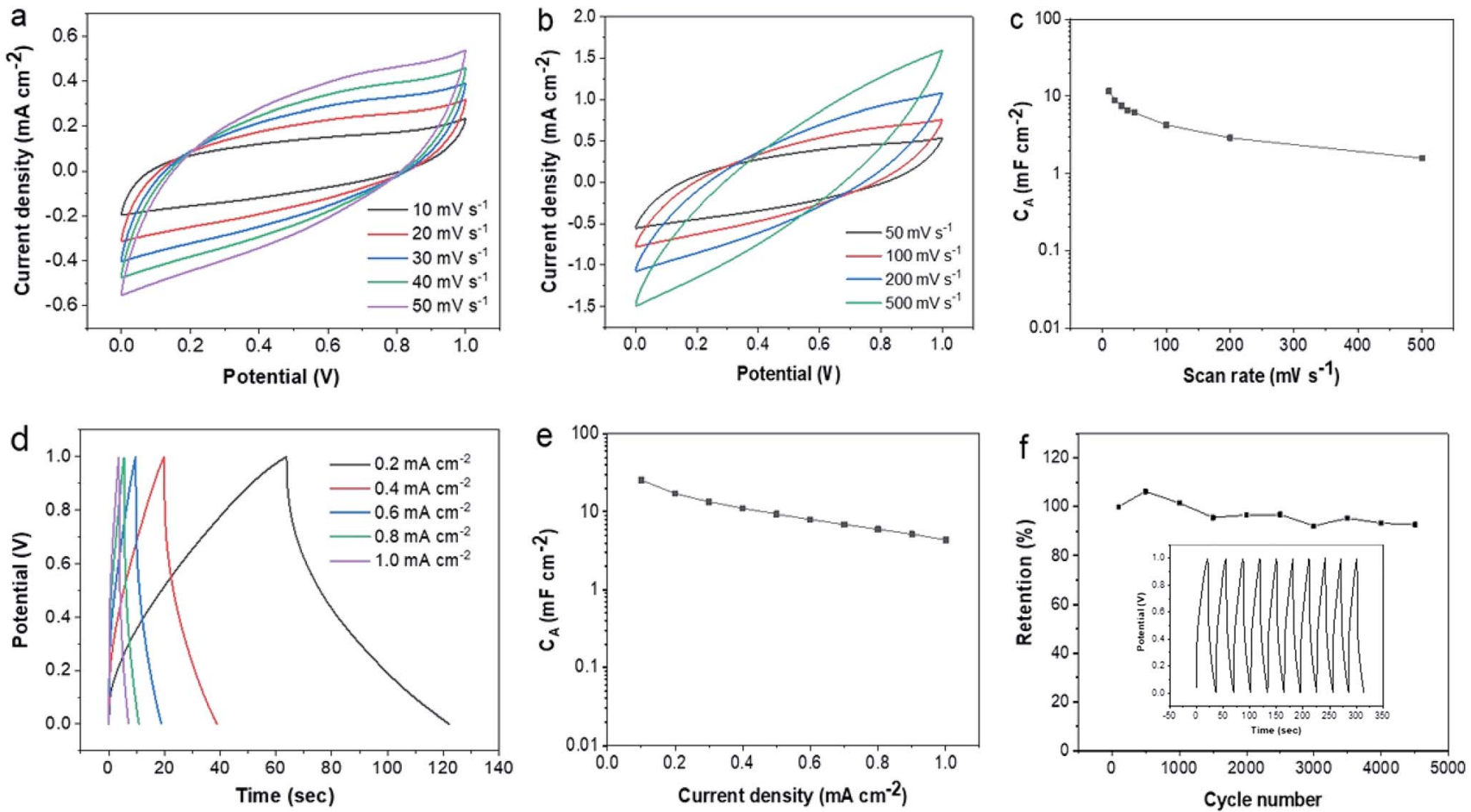

Fig. 5 Electrochemical performance of LIG-80-SC in $\mathrm{H}_{2} \mathrm{SO}_{4} / \mathrm{PVA}$ gel electrolyte. (a and b) CV curves at different scan rates (measured at a scan rate of $10-500 \mathrm{mV} \mathrm{s}^{-1}$ ). (c) $C_{A}$ calculated from CV curves as a function of scan rate. (d) $C D$ curves at various discharge current densities. (e) $C_{A}$ calculated from CD curves as a function of discharge current density. (f) Cycling stability during 4500 charge-discharge cycles at a current density of $0.2 \mathrm{~mA} \mathrm{~cm}^{-2}$; capacitance calculated from the CD curves at a current density of $0.2 \mathrm{~mA} \mathrm{~cm}^{-2}$; inset in (f) shows CD curves obtained for the first 10 cycles. 
example, both interdigitated and sandwiched SCs using pristine LIG from PI film and $1 \mathrm{M} \mathrm{PVA} / \mathrm{H}_{2} \mathrm{SO}_{4}$ gave about $8-9 \mathrm{mF} \mathrm{cm}{ }^{-2}$ at $0.5 \mathrm{~mA} \mathrm{~cm}{ }^{-2} \cdot{ }^{21,34}$ In the study reported by Zhang et al.,${ }^{25}$ ligninbased LIG coated with Au allowed interdigitated SCs with $1 \mathrm{M}$ $\mathrm{PVA} / \mathrm{H}_{2} \mathrm{SO}_{4}$ to reach maximal capacitance of $25.1 \mathrm{mF} \mathrm{cm}{ }^{-2}(6.27$ $\mathrm{F} \mathrm{cm}^{-3}$ ) at $0.01 \mathrm{~mA} \mathrm{~cm}^{-2}$, which was much higher than noncoated LIG electrode counterpart, but its capacitance decreased significantly down to $10 \mathrm{mF} \mathrm{cm}^{-2}$ at a higher current density of $0.5 \mathrm{~mA} \mathrm{~cm}^{-2}$. It remains unclear in their study why higher quality LIG with more porous structure did not stand out on electrochemical capacity. In contrast, the SC fabricated using pristine LIG derived from lignin in the present work was able to deliver comparable capacitance even at a ten-fold current density. If LIG-80 is coated with conductive materials, such as $\mathrm{Au}$ and polyaniline, it is reasonably expected that derived SC would have much higher capacitance. These findings suggested that different device architecture and high quality lignin-based LIG can lead to good performance of SCs.

In addition, the fabricated lignin-based LIG-SCs were evaluated for their energy and power densities. As seen from the Ragone plots (Fig. S7c and $d \dagger$ ), the device was able to deliver an $E_{\mathrm{A}}$ of $3.45 \mu \mathrm{W} \mathrm{h} \mathrm{cm}{ }^{-2}$ and an $E_{\mathrm{V}}$ of $0.651 \mathrm{~mW} \mathrm{~h} \mathrm{~cm}^{-3}$ at the current density of $0.1 \mathrm{~mA} \mathrm{~cm}{ }^{-2}$. Even with a higher current density $\left(0.5 \mathrm{~mA} \mathrm{~cm}{ }^{-2}\right)$, the $E_{\mathrm{A}}$ and $E_{\mathrm{V}}$ remained to be $1.16 \mu \mathrm{Wh}$

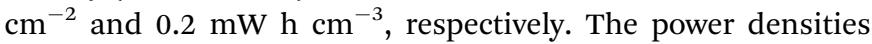
reached $0.418 \mathrm{~mW} \mathrm{~cm}{ }^{-2}\left(P_{\mathrm{A}}\right)$ and $78.86 \mathrm{~mW} \mathrm{~cm}^{-3}\left(P_{\mathrm{V}}\right)$ at a current density of $0.5 \mathrm{~mA} \mathrm{~cm}{ }^{-2}$, which are about half of that calculated at $1 \mathrm{~mA} \mathrm{~cm}^{-2}$. Compared to typical commercial SCs $\left(5.5 \mathrm{~V} / 100 \mathrm{mF}\right.$ ) and other LIG-based MSCs/SCs, ${ }^{17}$ our proposed device can offer comparable or even higher energy/power densities. The SC device also demonstrated good electrochemical stability. After 4500 cycles, more than $93 \%$ of the initial capacitance was retained (Fig. 5f). Overall, lignin-based LIG proved to be a highly promising active electrode material for energy storage application.

\section{Conclusion}

We have demonstrated a facile route to produce porous graphene from alkaline lignin using laser writing. Laser power was found to have significant effects on the formation of porous structure and degree of graphitization. By applying $80 \%$ of the laser power setting $(40 \mathrm{~W})$, hierarchical porous graphene was obtained from lignin film, which exhibited few-layered graphene with high crystallinity. LIG-80 demonstrated good electrochemical performance. The SC based on LIG-80 as both electrode and current collector delivered a high capacitance of $25.44 \mathrm{mF} \mathrm{cm}^{-2}$ (corresponding to $4.80 \mathrm{~F} \mathrm{~cm}^{-3}$ ), an energy density of $0.651 \mathrm{~mW} \mathrm{~h} \mathrm{~cm}{ }^{-3}$, and a power density of $148 \mathrm{~mW}$ $\mathrm{cm}^{-3}$. These findings suggested that lignin was successfully transformed into high quality porous LIG, which can be subsequently used for the fabrication of high-performance, solid-state SCs. This study opens a new avenue to costeffective lignin upgrading to value-added electrode materials. It would promote future research in using cheap technical lignin as carbon precursor for graphene fabrication. The simple laser writing process for lignin valorization toward energy storage devices shows great promise for low-cost, scalable application.

\section{Conflicts of interest}

The authors declare no conflict of interest.

\section{Acknowledgements}

We thank the support from the University of Missouri Electron Microscopy Core facility. We also thank Dr Steven Kelley at the Department of Chemistry in University of Missouri for his help with XRD analysis.

\section{References}

1 R. Rinaldi, R. Jastrzebski, M. T. Clough, J. Ralph, M. Kennema, P. C. A. Bruijnincx and B. M. Weckhuysen, Angew. Chem., Int. Ed., 2016, 55, 8164-8215.

2 A. J. Ragauskas, G. T. Beckham, M. J. Biddy, R. Chandra, F. Chen, M. F. Davis, B. H. Davison, R. A. Dixon, P. Gilna, M. Keller, P. Langan, A. K. Naskar, J. N. Saddler, T. J. Tschaplinski, G. A. Tuskan and C. E. Wyman, Science, 2014, 344, 1246843.

3 J. Zakzeski, P. C. Bruijnincx, A. L. Jongerius and B. M. Weckhuysen, Chem. Rev., 2010, 110, 3552-3599.

4 S. Laurichesse and L. Avérous, Prog. Polym. Sci., 2014, 39, 1266-1290.

5 S. Chatterjee and T. Saito, ChemSusChem, 2015, 8, 39413958.

6 S. Hu and Y.-L. Hsieh, RSC Adv., 2017, 7, 30459-30468.

7 N. Guo, M. Li, X. Sun, F. Wang and R. Yang, Green Chem., 2017, 19, 2595-2602.

8 Y. Song, J. Liu, K. Sun and W. Xu, RSC Adv., 2017, 7, 4832448332.

9 W. Zhang, M. Zhao, R. Liu, X. Wang and H. Lin, Colloids Surf., A, 2015, 484, 518-527.

10 C. Ma, Z. Li, J. Li, Q. Fan, L. Wu, J. Shi and Y. Song, Appl. Surf. Sci., 2018, 456, 568-576.

11 J. Yang, Y. Wang, J. Luo and L. Chen, ACS Omega, 2018, 3, 4647-4656.

12 J. W. Jeon, L. B. Zhang, J. L. Lutkenhaus, D. D. Laskar, J. P. Lemmon, D. Choi, M. I. Nandasiri, A. Hashmi, J. Xu, R. K. Motkuri, C. A. Fernandez, J. Liu, M. P. Tucker, P. B. McGrail, B. Yang and S. K. Nune, ChemSusChem, 2015, 8, 428-432.

13 H. C. Ho, N. A. Nguyen, K. M. Meek, D. M. Alonso, S. H. Hakim and A. K. Naskar, ChemSusChem, 2018, 11, 2953-2959.

14 R. Ye, D. K. James and J. M. Tour, Acc. Chem. Res., 2018, 51, 1609-1620.

15 R. Raccichini, A. Varzi, S. Passerini and B. Scrosati, Nat. Mater., 2015, 14, 271.

16 J. Lin, Z. Peng, Y. Liu, F. Ruiz-Zepeda, R. Ye, E. L. G. Samuel, M. J. Yacaman, B. I. Yakobson and J. M. Tour, Nat. Commun., 2014, 5, 5714. 
17 R. Ye, D. K. James and J. M. Tour, Adv. Mater., 2018, 31, 1803621.

18 M. F. El-Kady, V. Strong, S. Dubin and R. B. Kaner, Science, 2012, 335, 1326-1330.

19 C. Zhang, C. Zhang, Y. Xie, J.-W. Su, X. He, J. D. Demaree, M. H. Griep, J. L. Atwood and J. Lin, Chem.-Eur. J., 2019, 25, 4036-4039.

20 Y. Xie, C. Zhang, X. He, T. White, J. D. Demaree, M. Griep and J. Lin, J. Power Sources, 2018, 397, 37-43.

21 Z. Peng, J. Lin, R. Ye, E. L. G. Samuel and J. M. Tour, ACS Appl. Mater. Interfaces, 2015, 7, 3414-3419.

22 Y. Dong, S. C. Rismiller and J. Lin, Carbon, 2016, 104, 47-55. 23 Y. Chyan, R. Ye, Y. Li, S. P. Singh, C. J. Arnusch and J. M. Tour, ACS Nano, 2018, 12, 2176-2183.

24 R. Q. Ye, Y. Chyan, J. B. Zhang, Y. L. Li, X. Han, C. Kittrell and J. M. Tour, Adv. Mater., 2017, 29, 1702211.

25 W. L. Zhang, Y. J. Lei, F. W. Ming, Q. Jiang, P. M. F. J. Costa and H. N. Alshareef, Adv. Energy Mater., 2018, 8, 1600221.

26 L. Cançado, K. Takai, T. Enoki, M. Endo, Y. Kim, H. Mizusaki, A. Jorio, L. Coelho, R. Magalhaes-Paniago and M. J. A. P. L. Pimenta, Appl. Phys. Lett., 2006, 88, 163106.

27 Y. Gogotsi and P. Simon, Science, 2011, 334, 917-918.

28 V. Strauss, K. Marsh, M. D. Kowal, M. El-Kady and R. B. J. A. M. Kaner, Adv. Mater., 2018, 30, 1704449.
29 Z. Wang, D. Shen, C. Wu and S. Gu, Green Chem., 2018, 20, 5031-5057.

$30 \mathrm{~J} . \quad$ S. McDonald-Wharry, M. Manley-Harris and K. L. Pickering, Energy Fuels, 2016, 30, 7811-7826.

31 A. C. Ferrari, J. C. Meyer, V. Scardaci, C. Casiraghi, M. Lazzeri, F. Mauri, S. Piscanec, D. Jiang, K. S. Novoselov, S. Roth and A. K. Geim, Phys. Rev. Lett., 2006, 97, 187401.

32 S. Kwon, Y. Yoon, J. Ahn, H. Lim, G. Kim, J.-H. Kim, K.-B. Choi and J. J. C. Lee, Carbon, 2018, 137, 136-145.

33 S. Lee and S. Jeon, ACS Sustainable Chem. Eng., 2018, 7, 22702275.

34 L. Li, J. Zhang, Z. Peng, Y. Li, C. Gao, Y. Ji, R. Ye, N. D. Kim, Q. Zhong, Y. Yang, H. Fei, G. Ruan and J. M. Tour, Adv. Mater., 2016, 28, 838-845.

35 M. F. El-Kady and R. B. Kaner, Nat. Commun., 2013, 4, 1475. 36 W. Gao, N. Singh, L. Song, Z. Liu, A. L. M. Reddy, L. J. Ci, R. Vajtai, Q. Zhang, B. Q. Wei and P. M. Ajayan, Nat. Nanotechnol., 2011, 6, 496-500.

37 D. Pech, M. Brunet, H. Durou, P. Huang, V. Mochalin, Y. Gogotsi, P.-L. Taberna and P. Simon, Nat. Nanotechnol., 2010, 5, 651.

38 Z. S. Wu, K. Parvez, X. Feng and K. Müllen, Nat. Commun., 2013, 4, 2487. 